\title{
GLOBAL WELL-POSEDNESS AND SCATTERING FOR THE QUANTUM ZAKHAROV SYSTEM IN $L^{2}$
}

\author{
YUNG-FU FANG AND KENJI NAKANISHI \\ (Communicated by Joachim Krieger)
}

\begin{abstract}
We study the Cauchy problem for the quantum Zakharov system in the class of square-integrable functions on the Euclidean space of general dimensions. The local well-posedness is proven for dimensions up to eight, together with global existence for dimensions up to five, as well as scattering for small initial data in dimensions greater than three.
\end{abstract}

\section{INTRODUCTION}

In this paper, we consider the global well-posedness and scattering of solutions in $L^{2}\left(\mathbb{R}^{d}\right)$ for the quantum Zakharov (QZ) system in general space dimensions $d \in \mathbb{N}$. The system reads as

$$
\left\{\begin{array}{l}
i E_{t}+\Delta E-\Delta^{2} E=n E, \quad(t, x) \in \mathbb{R} \times \mathbb{R}^{d} \\
n_{t t}-\Delta n+\Delta^{2} n=\Delta|E|^{2} \\
E(0)=E_{0}, n(0)=n_{0}, \quad \partial_{t} n(0)=n_{1}, \quad x \in \mathbb{R}^{d}
\end{array}\right.
$$

where $E(t, x): \mathbb{R}^{1+d} \rightarrow \mathbb{C}$ and $n(t, x): \mathbb{R}^{1+d} \rightarrow \mathbb{R}$ are the unknown quantities. See [8, [17], and [18] for the physical meanings. All the coefficients are set to 1 in this paper, since their sizes play no role in our analysis, nor do the signs of the quadratic terms on the right side. In other words, one can change their sizes, as well as the signs on the right side, in (1.1) without changing the conclusions. It should be noted, however, that our estimates are not uniform with respect to the size.

When the quantum effect is absent, the system is reduced to the classical Zakharov system

$$
\left\{\begin{array}{l}
i E_{t}+\Delta E=n E, \quad(t, x) \in \mathbb{R} \times \mathbb{R}^{d} \\
n_{t t}-\Delta n=\Delta|E|^{2} \\
E(0)=E_{0}, n(0)=n_{0}, \partial_{t} n(0)=n_{1}, \quad x \in \mathbb{R}^{d} .
\end{array}\right.
$$

The regular solutions of (1.2) satisfy the conservation of mass

$$
\int_{\mathbb{R}^{d}}|E(t)|^{2} d x=\int_{\mathbb{R}^{d}}|E(0)|^{2} d x
$$

Received by the editors December 23, 2018.

2010 Mathematics Subject Classification. Primary 35L30; Secondary 35L05, 35Q55.

Key words and phrases. quantum Zakharov system, well-posedness, global solutions, scatterng.

The first author was partially supported by MOST, MRPC, and NCTS (Taiwan).

The second author was supported by JSPS KAKENHI Grant Number JP17H02854.

The first author is the corresponding author.

(C)2019 by the authors under Creative Commons Attribution-Noncommercial 3.0 License (CC BY NC 3.0) 
as well as the conservation of the Hamiltonian

$$
\|\nabla E\|_{L^{2}}^{2}+\frac{1}{2}\left(\left\|n_{t}\right\|_{\dot{H}^{-1}}^{2}+\|n\|_{L^{2}}^{2}\right)+\int_{\mathbb{R}^{d}} n|E|^{2} d x .
$$

Analogously, (1.1) has the conservation of mass

$$
\int_{\mathbb{R}^{d}}|E(t)|^{2} d x=\int_{\mathbb{R}^{d}}|E(0)|^{2} d x
$$

as well as the conservation of the Hamiltonian

$$
\|E\|_{L^{2}}^{2}+\|\Delta E\|_{L^{2}}^{2}+\frac{1}{2}\left(\left\|n_{t}\right\|_{\dot{H}^{-1}}^{2}+\|n\|_{L^{2}}^{2}+\|\nabla n\|_{L^{2}}^{2}\right)+\int_{\mathbb{R}^{d}} n|E|^{2} d x
$$

although we will not use the Hamiltonian structure in this paper, since our solutions will be merely in $L^{2}\left(\mathbb{R}^{d}\right)$. Note that to have a positive definite quadratic part in the Hamiltonian, the signs of the two quadratic terms in (1.1) should be the same, but we do not need it in this paper.

For simplicity, we transform (1.1) into the first order equations in $t$ by the change of variable

$$
\mathrm{H}:=\sqrt{-\Delta+\Delta^{2}}, \quad \mathcal{N}:=n+i \mathrm{H}^{-1} \partial_{t} n, \quad n=\operatorname{Re} \mathcal{N},
$$

where $\operatorname{Re} \mathcal{N}$ is the real part of $\mathcal{N}$. Thus the quantum Zakharov system (1.1) becomes

$$
\left\{\begin{array}{l}
i E_{t}-\mathrm{H}^{2} E=(\operatorname{Re} \mathcal{N}) E, \quad(t, x) \in \mathbb{R} \times \mathbb{R}^{d} ; \\
i \partial_{t} \mathcal{N}-\mathrm{H} \mathcal{N}=-\Delta \mathrm{H}^{-1}|E|^{2} ; \\
E(0)=E_{0}, \quad \mathcal{N}(0)=\mathcal{N}_{0}, \quad x \in \mathbb{R}^{d}
\end{array}\right.
$$

where $\mathcal{N}_{0}:=n_{0}+i \mathrm{H}^{-1} n_{1}$.

In this paper, we study the Cauchy problem for (1.6) with initial data $\left(E_{0}, \mathcal{N}_{0}\right) \in$ $L^{2}\left(\mathbb{R}^{d}\right) \times L^{2}\left(\mathbb{R}^{d}\right)$, locally and globally in time, just using the classical Strichartz estimates. In most of the preceding works, the initial data (and so the solutions) with more regularity were considered, except some recent results [5, 7] in one dimension. It is needless to say that $L^{2}$ is the most important and basic function space in mathematics, and it is convenient to work with solutions with no derivative for various reasons, including numerical computations. The $L^{2}$ norm is physically important for the Zakharov (type) systems, as it measures the total electric energy of the plasma. For the mathematical analysis of PDE, it is important to solve the system in the invariant (conserved) function space, particularly for global analysis of the solutions. In comparison with the classical Zakharov system (1.2), it is interesting to see how much the system (or its dynamics) is tamed by the quantum effect. It turns out that we can deal with the $L^{2}$ data in high space dimensions as well, unlike the classical case, where the well-posedness for $E_{0} \in L^{2}\left(\mathbb{R}^{d}\right)$ is known [2, 6, 13. only for $d=1,2$, and the solution can blow up in $d=2,9,10$. It is interesting because tremendous efforts have been devoted to constructing and analyzing rough solutions to nonlinear dispersive equations, where models arising in the mathematical physics tend to exhibit various mathematical challenges, making the corresponding analysis complicated even if the equations look very simple. This paper suggests that including some more physical effects in the equations can make our mathematical understanding much easier and better.

The main results of this paper are as follows.

Theorem 1.1 (Well-posedness). For $1 \leq d \leq 8$, the quantum Zakharov system (1.6) is locally well-posed in $L^{2}\left(\mathbb{R}^{d}\right) \times L^{2}\left(\mathbb{R}^{d}\right)$. Moreover, if $d \leq 5$, then all the 
solutions are global in time, and there exist positive constants $\alpha, \beta \in(0,1), p \in$ $[2, \infty)$, and $C \in(0, \infty)$, dependent only on $d$, such that

$\|E(t)\|_{L^{2}}=\|E(0)\|_{L^{2}}<\infty, \quad\|\mathcal{N}(t)\|_{L^{2}} \leq C\|\mathcal{N}(0)\|_{L^{2}}+C|t|^{\alpha}\left\|E_{0}\right\|_{L^{2}}^{2}+C|t|^{\beta}\left\|E_{0}\right\|_{L^{2}}^{p}$.

A more precise form of the upper bound is given in the proof for each $d$; see (5.4).

Theorem 1.2 (Small data scattering). Let $4 \leq d \leq 8$. Assume that $\left(E_{0}, \mathcal{N}_{0}\right) \in$ $L^{2}\left(\mathbb{R}^{d}\right) \times L^{2}\left(\mathbb{R}^{d}\right)$ is sufficiently small in $L^{2}$-norm. Then the solution $(E, \mathcal{N})$ of (1.6) is global in time, and there exist $\left(E_{ \pm}, \mathcal{N}_{ \pm}\right) \in L^{2}\left(\mathbb{R}^{d}\right) \times L^{2}\left(\mathbb{R}^{d}\right)$ such that

$$
\left\|E(t)-e^{-i t \mathrm{H}^{2}} E_{ \pm}\right\|_{L^{2}}+\left\|\mathcal{N}(t)-e^{-i t \mathrm{H}} \mathcal{N}_{ \pm}\right\|_{L^{2}} \rightarrow 0 \quad(t \rightarrow \pm \infty) .
$$

In short, the solution $(E, \mathcal{N})$ scatters in $L^{2}\left(\mathbb{R}^{d}\right) \times L^{2}\left(\mathbb{R}^{d}\right)$ as $t \rightarrow \pm \infty$.

Remark 1. It is worth noting that we do not need at all the dispersive nature of the (fourth order) wave equation, either for the small data scattering (Theorem 1.2) or for the local well-posedness in $d \leq 7$ (Theorem[1.1). It means that $\mathrm{H}$ in the left side of the second equation of (1.6) could be replaced with any self-adjoint operator on $L^{2}$ for those results.

The outline of the paper is as follows. In Section 2, we prepare some notation and definitions. In Section 3, we recall the Strichartz estimates for the linear equations, which is the main tool for analysis in this paper. In Section 4 , we prove the local well-posedness result, Theorem 1.1. In Section 5, we show the global well-posedness and polynomial bound of wave, Theorem 1.1. Finally in Section 6. we prove the scattering result, Theorem 1.2 .

\section{Notation and Duhamel formulae}

Let us denote

$$
\mathcal{D}:=\sqrt{-\Delta}, \quad\langle\mathcal{D}\rangle:=\sqrt{1-\Delta}, \quad \mathrm{U}:=\mathcal{D}\langle\mathcal{D}\rangle^{-1} .
$$

Then we have $\mathrm{H}=\mathcal{D}\langle\mathcal{D}\rangle$. We also denote the Fourier transform of $u(x)$ over $x \in \mathbb{R}^{d}$ by $\mathcal{F} u(\xi)$.

For $s \in \mathbb{R}$, we denote by $H^{s}\left(\mathbb{R}^{d}\right)$ and $\dot{H}^{s}\left(\mathbb{R}^{d}\right)$ the usual inhomogeneous and homogeneous Sobolev spaces equipped with the norms, respectively,

$\|u\|_{H^{s}}:=\left\|\langle\mathcal{D}\rangle^{s} u\right\|_{L^{2}}=\left\|\left(1+|\xi|^{2}\right)^{\frac{s}{2}} \mathcal{F} u\right\|_{L^{2}} \quad$ and $\quad\|u\|_{\dot{H}^{s}}:=\left\|\mathcal{D}^{s} u\right\|_{L^{2}}=\left\||\xi|^{s} \mathcal{F} u\right\|_{L^{2}}$.

For $p, q \in[1, \infty]$ and $s \in \mathbb{R}, B_{p, q}^{s}\left(\mathbb{R}^{d}\right)$ denotes the inhomogeneous Besov space equipped with the norm

$$
\|u\|_{B_{p, q}^{s}}=\left\|2^{j s}\right\| \mathcal{F}^{-1} \psi_{j}(\xi) \mathcal{F} u\left\|_{L^{p}\left(x \in \mathbb{R}^{d}\right)}\right\|_{\ell^{q}(j \geq 0)},
$$

where $\psi_{j}(\xi):=\psi\left(2^{-j} \xi\right)-\psi\left(2^{-j+1} \xi\right)$ and $\psi_{0}:=\psi$, with a fixed smooth and radial function $\psi: \mathbb{R}^{d} \rightarrow \mathbb{R}$ satisfying $\psi(\xi)=1$ for $|\xi| \leq 1,0$ for $|\xi| \geq 2$. Similarly, the homogeneous Besov space is denoted by $\dot{B}_{p, q}^{s}\left(\mathbb{R}^{d}\right)$.

For any $T \in(0, \infty]$ or any interval $I \subset \mathbb{R}$ and any Banach space $X$ of functions on $\mathbb{R}^{d}$, the $X$-valued $L^{p}$ norm in time is denoted by

$$
\|u\|_{L_{T}^{p} X}:=\left[\int_{0}^{T}\|u(t)\|_{X}^{p} d t\right]^{1 / p}, \quad\|u\|_{L_{t \in I}^{p} X}:=\left[\int_{I}\|u(t)\|_{X}^{p} d t\right]^{1 / p},
$$


for any $1 \leq p<\infty$, and similarly for $p=\infty$. We often add the subscript $X_{x}=X$ in order to highlight the function space for $x \in \mathbb{R}^{d}$.

The complex interpolation space and the real interpolation space are denoted respectively by $\left[X_{0}, X_{1}\right]_{\theta}$ and $\left(X_{0}, X_{1}\right)_{\theta, p}$, for a couple of Banach spaces $\left(X_{0}, X_{1}\right)$, $\theta \in[0,1]$, and $p \in[1, \infty]$.

For the fourth order Schrödinger equation of $E: \mathbb{R}^{1+d} \rightarrow \mathbb{C}$,

$$
i E_{t}-\mathrm{H}^{2} E=F,
$$

we have the Duhamel formula

$$
E(t)=e^{-i t \mathrm{H}^{2}} E(0)-i \int_{0}^{t} e^{-i(t-s) \mathrm{H}^{2}} F(s) d s .
$$

For the (square-root of) fourth order wave equation to $\mathcal{N}: \mathbb{R}^{1+d} \rightarrow \mathbb{C}$,

$$
i \partial_{t} \mathcal{N}-\mathrm{H} \mathcal{N}=G
$$

we have the Duhamel formula

$$
\mathcal{N}(t)=e^{-i t \mathrm{H}} \mathcal{N}(0)-i \int_{0}^{t} e^{-i(t-s) \mathrm{H}} G(s) d s .
$$

\section{Strichartz estimates}

In this section, we recall the Strichartz estimates for the operators $\mathrm{H}$ and $\mathrm{H}^{2}$, which follow from the standard arguments by the Fourier analysis. Following 25, a pair $(q, r)$ is called Schrödinger admissible, for short $S$-admissible, if

$$
2 \leq q, r \leq \infty, \quad(q, r, d) \neq(2, \infty, 2), \quad \frac{2}{q}+\frac{d}{r}=\frac{d}{2} .
$$

A pair $(q, r)$ is called biharmonic admissible, for short $B$-admissible, if

$$
2 \leq q, r \leq \infty, \quad(q, r, d) \neq(2, \infty, 4), \quad \frac{4}{q}+\frac{d}{r}=\frac{d}{2} .
$$

Lemma 1 (Pausader [25]). Let $E \in C\left([0, T], H^{-4}\left(\mathbb{R}^{d}\right)\right)$ be a solution of (2.3). For any $B$-admissible pairs $\left(q_{1}, r_{1}\right)$ and $\left(q_{2}, r_{2}\right)$, it satisfies

$$
\|E\|_{L_{T}^{q_{1}} L_{x}^{r_{1}\left(\mathbb{R}^{d}\right)}} \lesssim\|E(0)\|_{L_{x}^{2}\left(\mathbb{R}^{d}\right)}+\|F\|_{L_{T}^{q_{2}{ }^{\prime}} L_{x}^{r_{2}{ }^{\prime}}\left(\mathbb{R}^{d}\right)},
$$

and for any $S$-admissible pairs $\left(q_{1}, r_{1}\right)$ and $\left(q_{2}, r_{2}\right)$ and any $s \in \mathbb{R}$,

$$
\|E\|_{L_{T}^{q_{1}} B_{r_{1}, 2}^{s+2 / q_{1}}\left(\mathbb{R}^{d}\right)} \lesssim\|E(0)\|_{H^{s}\left(\mathbb{R}^{d}\right)}+\|F\|_{L_{T}^{q_{2}{ }^{\prime}} B_{r_{2}^{\prime}, 2}^{s-2 / q_{2}}\left(\mathbb{R}^{d}\right)} .
$$

In both estimates, the implicit constants depend only on $d, q_{1}, q_{2}$.

The proof is based on the work of Kenig-Ponce-Vega 20 or the works of BenArtzi-Koch-Saut 3], Pausader [25, and Keel-Tao [19], together with some modifications. We only sketch the proof.

Proof. We consider the integral

$$
e^{i t \phi(\mathcal{D})} f=\int_{\mathbb{R}^{d}} e^{i t \phi(|\xi|)+i \xi x} \hat{f}(\xi) d \xi,
$$

where the phase function is given by $\phi(r)=r^{2}+r^{4}$. Then we have

$$
\phi^{\prime}(r) \sim r\langle r\rangle^{2} \text { and } \phi^{\prime \prime}(r) \sim\langle r\rangle^{2} .
$$


The Hessian of the phase function $\phi$ can be expressed in the polar coordinate $\xi=r \omega \in \mathbb{R}^{d}(r=|\xi|)$ as follows (for $\left.\xi \neq 0\right)$ :

$$
\nabla^{2} \phi(r)=\nabla\left(\phi^{\prime}(r) \omega\right)=\phi^{\prime \prime}(r) \omega^{*} \omega+\frac{\phi^{\prime}(r)}{r}\left(I-\omega^{*} \omega\right),
$$

where $\omega^{*} \omega$ is the projection onto $\operatorname{span}\{\xi\}$ and $I-\omega^{*} \omega$ is the orthogonal projection onto $\{\xi\}^{\perp}$. Note that $\operatorname{span}\{\xi\}$ is one dimensional and $\{\xi\}^{\perp}$ is $(d-1)$ dimensional. Hence we have

$$
\sqrt{\operatorname{det} \nabla^{2} \phi} \sim\langle r\rangle^{d}
$$

as well as $\left|\nabla^{\alpha} \phi(r)\right| \lesssim\left|\phi^{\prime}(r)\right| r^{1-|\alpha|}$ for all multi-index $\alpha$ such that $|\alpha| \geq 2$. Then the decay estimate can be obtained by the stationary phase method as follows (cf. [25, (3.6)] or [11, Theorem 2.2]):

$$
\left\|e^{-i t \mathrm{H}^{2}} f\right\|_{B_{\infty, 2}^{0}} \lesssim|t|^{-\frac{d}{2}}\|f\|_{B_{1,2}^{-d}}
$$

Via duality argument and $T T^{*}$ argument (cf. [19]), we obtain the Strichartz estimate (3.4) for $S$-admissible exponents. Applying the Sobolev embedding to both sides of the estimate, we obtain (3.3) for $B$-admissible pairs unless $r_{1}$ or $r_{2}$ is $\infty$. The last case is covered by the interpolation inequality as follows. If $(q, \infty)$ is $B$ admissible, then $q=8 / d>2$ and $(q, 4)$ is $S$-admissible. Hence there exists small $\varepsilon>0$ such that the pairs of exponents $\left(q_{ \pm}, 4_{ \pm}\right)$defined by

$$
2 / q_{ \pm}=2 / q \pm \varepsilon, \quad d / 4_{ \pm}=d / 4 \mp \varepsilon
$$

are $S$-admissible. Note that the pairs $\left(q_{ \pm}, 4_{ \pm}\right)$are near the pair $(q, 4)$. Then the Sobolev embeddings and the real interpolation, i.e.,

$$
B_{4_{ \pm}, 2}^{2 / q_{ \pm}} \subset B_{\infty, 2}^{ \pm 2 \varepsilon} \quad \text { and } \quad\left(B_{\infty, 2}^{2 \varepsilon}, B_{\infty, 2}^{-2 \varepsilon}\right)_{1 / 2,1}=B_{\infty, 1}^{0} \subset L^{\infty}
$$

imply the interpolation inequality $\|u\|_{L^{\infty}} \lesssim\|u\|_{B_{4_{+}, 2}^{2 / q_{+}}}^{1 / 2}\|u\|_{B_{4_{-}, 2}^{2 / q_{-}}}^{1 / 2}$, and by Hölder in $t$,

$$
\|u\|_{L_{T}^{q} L_{x}^{\infty}} \lesssim\|u\|_{L_{T}^{q_{+} B_{4_{+}, 2}^{2 / q_{+}}}}^{1 / 2}\|u\|_{L_{T}^{q_{-} B_{4_{-}, 2}^{2 / q_{-}}}}^{1 / 2} \lesssim\|u\|_{L_{T}^{q_{+}} B_{4_{+}, 2}^{2 / q_{+}} \cap L_{T}^{q_{-}} B_{4_{-}, 2}^{2 / q_{-}}} .
$$

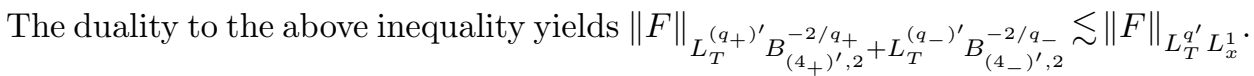
Thus the cases with $r_{1}=\infty$ or $r_{2}=\infty$ follow from the estimate for the corresponding pairs of $S$-admissible exponents.

Remark 2. We supplement the proof of the Strichartz estimate (3.3) in the case of $r=\infty$ given in [25].

Lemma 2 (Gustafson-Nakanishi-Tsai [11, Theorem 2.1]). Let $\mathcal{N}$ be a solution of (2.5). If $\left(q_{i}, r_{i}\right)$ are $S$-admissible for $i=1,2$, we have, with $\gamma_{i}:=(1-2 / d) / q_{i}$,

$$
\left\|\mathrm{U}^{-\gamma_{1}} \mathcal{N}\right\|_{L_{T}^{q_{1}} L_{x}^{r_{1}}\left(\mathbb{R}^{d}\right)} \lesssim\left\|\mathcal{N}_{0}\right\|_{L^{2}\left(\mathbb{R}^{d}\right)}+\left\|\mathrm{U}^{\gamma_{2}} G\right\|_{L_{T}^{q_{2}^{\prime}} L_{x}^{r_{2}^{\prime}\left(\mathbb{R}^{d}\right)}},
$$

where the implicit constant depends only on $d, q_{1}, q_{2}$.

Note that $\mathrm{U}^{\alpha}$ is bounded on $L^{p}\left(\mathbb{R}^{d}\right)$ for any $\alpha \geq 0$ and $p \in[1, \infty]$. The Strichartz estimate gains a positive power of $\mathrm{U}$ for $d \geq 3$, but we do not use it in this paper. On the other hand, it suffers from a negative power of $\mathrm{U}$ in $d=1$, against which we exploit $U$ in the equation acting on $|E|^{2}$.

For the proof of the above lemma, the reader is referred to the work of GustafsonNakanishi-Tsai [1]. Here we only sketch the proof. 
Proof. We consider $e^{i t \phi(\mathcal{D})} f$ with the phase function given by $\phi(r)=r\langle r\rangle$. Then we have

$$
\phi^{\prime}(r) \sim\langle r\rangle \quad \text { and } \quad \phi^{\prime \prime}(r) \sim \frac{r}{\langle r\rangle} .
$$

The Hessian of the phase function $\phi$ can be expressed as (3.5). Hence we have

$$
\sqrt{\operatorname{det} \nabla^{2} \phi} \sim\left(\frac{r}{\langle r\rangle}\right)^{-\frac{d-2}{2}}
$$

as well as $\left|\nabla^{\alpha} \phi(r)\right| \lesssim\left|\phi^{\prime}(r)\right| r^{1-|\alpha|}$. Hence the decay estimate can be obtained from the stationary phase method as follows:

$$
\left\|e^{i t \phi(\mathcal{D})} f\right\|_{\dot{B}_{\infty, 2}^{0}} \lesssim|t|^{-\frac{d}{2}}\left\|\mathrm{U}^{\frac{d-2}{2}} f\right\|_{\dot{B}_{1,2}^{0}} .
$$

Via duality argument and $T T^{*}$ argument, as well as the embedding $\dot{B}_{r, 2}^{0} \subset L^{r}$ for $r \in[2, \infty)$, we obtain the Strichartz estimate (3.6) unless $r_{1}=\infty$ or $r_{2}=\infty$. The last case happens only in the case of $d=1$, where we can use the van der Corput lemma (see [26]) to obtain

$$
\sup _{x \in \mathbb{R}}\left|\int_{\mathbb{R}} e^{-i t \phi(\xi)+i x \xi} \mathrm{U}(\xi)^{1 / 2} \chi(\xi) d \xi\right| \lesssim|t|^{-1 / 2}\left(\|\chi\|_{L^{\infty}}+\left\|\chi^{\prime}\right\|_{L^{1}}\right),
$$

for any $\chi \in C^{1}(\mathbb{R})$ compactly supported in $\mathbb{R} \backslash\{0\}$, where $\mathrm{U}(\xi):=|\xi| /\langle\xi\rangle$. Applying the above estimate to $\chi_{N}:=\mathcal{F}^{-1}[\psi(\xi / N)-\psi(N \xi)]$, we obtain $\left\|e^{-i t \mathrm{H}} \mathrm{U}^{1 / 2} \chi_{N}\right\|_{L^{\infty}}$ $\lesssim|t|^{-1 / 2}$ uniformly for $N \geq 1$. Since $\chi_{N} * f \rightarrow f$ as $N \rightarrow \infty$ in the distribution sense for any $f \in L^{1}$, we obtain

$$
\begin{aligned}
\left\|e^{-i t \mathrm{H}} \mathrm{U}^{1 / 4} f\right\|_{L^{\infty}(\mathbb{R})} & \leq \varlimsup_{N \rightarrow \infty}\left\|\left(e^{-i t \mathrm{H}} \mathrm{U}^{1 / 2} \chi_{N}\right) *\left(\mathrm{U}^{-1 / 4} f\right)\right\|_{L^{\infty}} \\
& \lesssim|t|^{-1 / 2}\left\|\mathrm{U}^{-1 / 4} f\right\|_{L^{1}} .
\end{aligned}
$$

Using this decay estimate together with the duality and $T T^{*}$ arguments, we obtain the Strichartz estimate (3.6) with $r_{1}, r_{2} \in\{2, \infty\}$. The remaining cases are covered by the complex interpolation.

To prove (3.7), fix any $t>0, x \in \mathbb{R}$ and let $\theta(\xi):=-t \phi(\xi)+x \xi$ be the phase function. Since $\theta^{\prime \prime}=t \phi^{\prime \prime} \sim t \mathrm{U}(\xi)$, there exists at most one $\xi_{0} \in \mathbb{R}$ such that $\theta^{\prime}\left(\xi_{0}\right)=0$, namely, $\phi^{\prime}\left(\xi_{0}\right)=x / t$. Using smooth dyadic decomposition, we can decompose $\chi=\chi_{1}+\chi_{2}+\chi_{3}$ with some $\chi_{j} \in C^{1}(\mathbb{R})$ satisfying:

(1) $|\xi| \gtrsim 1$ on $\operatorname{supp} \chi_{1}$.

(2) $\xi \sim \xi_{0}$ on $\operatorname{supp} \chi_{2}$.

(3) $\xi \nsim \xi_{0}$ and $|\xi| \lesssim 1$ on $\operatorname{supp} \chi_{3}$.

(4) $\operatorname{supp} \chi_{j} \subset \operatorname{supp} \chi$ and $\left\|\chi_{j}\right\|_{L^{\infty}}+\left\|\chi_{j}^{\prime}\right\|_{L^{1}} \lesssim\|\chi\|_{L^{\infty}}+\left\|\chi^{\prime}\right\|_{L^{1}}$ for all $j=1,2,3$.

Let $I_{j}:=\int_{\mathbb{R}} e^{i \theta} \mathrm{U}^{1 / 2} \chi_{j} d \xi$ be the corresponding decomposition of the integral. The desired bound on $I_{1}, I_{2}$ follows from the van der Corput lemma with the second derivative $\theta^{\prime \prime}=-t \phi^{\prime \prime}<0$, using $\phi^{\prime \prime} \gtrsim 1$ on $\operatorname{supp} \chi_{1}$ and $\phi^{\prime \prime} \sim \mathrm{U}(\xi) \sim \mathrm{U}\left(\xi_{0}\right)$ on $\operatorname{supp} \chi_{2}$. On $I_{3}$ we apply the lemma with the first derivative $\left|\theta^{\prime}\right| \sim\left|t \phi^{\prime}\right| \sim|t|$ on $\operatorname{supp} \chi_{3}$, which yields $\left|I_{3}\right| \lesssim|t|^{-1}\left(\left\|\chi_{3}\right\|_{L^{\infty}}+\left\|\chi_{3}^{\prime}\right\|_{L^{1}}\right)$. Interpolation with the trivial bound $\left|I_{3}\right| \lesssim\left\|\chi_{3}\right\|_{L^{\infty}}$ yields the desired bound on $I_{3}$ and thus (3.7).

Remark 3. The Stricharz estimate given in 11] is for the dimension $d \geq 2$. We complete the proof of the Strichartz estimate (3.6) for all dimensions. 


\section{LOCAL WELL-POSEDNESS FOR THE $Q Z$ SYSTEM}

In this section, we prove the local well-posedness for (1.6) in $L^{2}\left(\mathbb{R}^{d}\right)$ for $1 \leq d \leq 8$, which is the first part of Theorem 1.1. The second part of the theorem, namely, the global well-posedness for $d \leq 5$, will be proven in the next section. To prove the local well-posedness, we define the maps for the Duhamel part in (2.4) and (2.6):

$$
\begin{gathered}
\Phi(E, \mathcal{N})(t)=-i \int_{0}^{t} e^{-i(t-s) \mathrm{H}^{2}}((\operatorname{Re} \mathcal{N}) E)(s) d s \\
\Psi(E)(t)=-i \int_{0}^{t} e^{-i(t-s) \mathrm{H}} \mathrm{U}|E(s)|^{2} d s
\end{gathered}
$$

for $(E, \mathcal{N}) \in C\left([0, T] ;\left(L^{2}\left(\mathbb{R}^{d}\right)\right)^{2}\right)$. The free solutions for $\left(E_{0}, N_{0}\right) \in\left(L^{2}\left(\mathbb{R}^{d}\right)\right)^{2}$ are denoted by

$$
E_{\mathrm{F}}:=e^{-i t \mathrm{H}^{2}} E_{0}, \quad \mathcal{N}_{\mathrm{F}}:=e^{-i t \mathrm{H}} \mathcal{N}_{0} .
$$

Then the iteration map $\Lambda$ is defined by

$$
\Lambda(E, \mathcal{N})(t):=\left(E_{\mathrm{F}}+\Phi(E, \mathcal{N}), \mathcal{N}_{\mathrm{F}}+\Psi(E)\right)(t) .
$$

For the sake of convenience, we denote some special exponents by

$$
q_{1}:=\frac{8}{d}, \quad \tilde{q}_{1}:=\frac{16}{d}, \quad q_{2}:=\frac{4}{d-2}, \quad \tilde{q}_{2}:=\frac{8}{d-2}, \quad 2\{s\}:=\frac{2 d}{d+2 s}
$$

for any $s \in(-d / 2, d / 2)$. The last notation is linked to the Sobolev embedding:

$$
0 \leq s<d / 2 \Longrightarrow \dot{H}^{s} \subset L^{2\{-s\}}, \quad L^{2\{s\}} \subset \dot{H}^{-s} .
$$

Notice that the pairs $\left(q_{1}, 4\right)$ for $d \leq 4,\left(q_{2}, d\right)$ for $2 \leq d \leq 4$, and $(2,2\{-1\})$ for $d>2$ are $S$-admissible, while the pairs $\left(\tilde{q}_{1}, 4\right)$ for $d \leq 8,\left(\tilde{q}_{2}, d\right)$ for $2 \leq d \leq 6$, and $(2,2\{-2\})$ for $d>4$ are $B$-admissible.

Let $S$ and $W$ be Banach spaces in $C_{t}\left([0, T] ; L_{x}^{2}\right)$, with the Strichartz norms given by Lemma 1 and Lemma 2, respectively, for $L_{x}^{2}$ initial data, defined by the norm

$$
\|u\|_{S}:= \begin{cases}\|u\|_{L_{T}^{\infty} L_{x}^{2}}+\|u\|_{L_{T}^{4} B_{\infty, 2}^{1 / 2}} & (d=1), \\ \|u\|_{L_{T}^{\infty} L_{x}^{2}}+\|u\|_{L_{T}^{2 / \theta} B_{2\{-\theta\}, 2}^{\theta}} & (d=2), \\ \|u\|_{L_{T}^{\infty} L_{x}^{2}}+\|u\|_{L_{T}^{2} B_{2\{-1\}, 2}^{1}} & (d \geq 3),\end{cases}
$$

where $\theta \in[1 / 2,1)$ is arbitrarily fixed and

$$
\|u\|_{W}:= \begin{cases}\|u\|_{L_{T}^{\infty} L_{x}^{2}}+\left\|\mathrm{U}^{-1 / 4} u\right\|_{L_{T}^{4} L_{x}^{\infty}} & (d=1), \\ \|u\|_{L_{T}^{\infty} L_{x}^{2}}+\|u\|_{L_{T}^{2 / \theta} L_{x}^{2\{-\theta\}}} & (d=2), \\ \|u\|_{L_{T}^{\infty} L_{x}^{2}}+\|u\|_{L_{t}^{2} L_{x}^{2\{-1\}}} & (d \geq 3) .\end{cases}
$$

Note that the second components in $d=2$ are the complex interpolation spaces between $L_{T}^{\infty} L_{x}^{2}$ and the prohibited endpoint space corresponding to the case $\theta=1$.

We are going to prove that the map $\Lambda$ is a contraction on a closed subset of $S \times W$ equipped with the norm $\|(E, \mathcal{N})\|_{S \times W}=\|E\|_{S}+\|\mathcal{N}\|_{W}$. First, the Strichartz estimates (3.4) and (3.6) imply that

$$
\left\|\left(E_{\mathrm{F}}, \mathcal{N}_{\mathrm{F}}\right)\right\|_{S \times W} \lesssim\left\|E_{0}\right\|_{L_{x}^{2}}+\left\|\mathcal{N}_{0}\right\|_{L_{x}^{2}} .
$$


Hence the main task to prove the local well-posedness in Theorem 1.1 is to control the Duhamel terms $\Phi(E, \mathcal{N})$ and $\Psi(E)$, respectively, in $S$ and $W$, which can be done via using the same Strichartz estimates.

Proof of Theorem 1.1 (Local well-posedness). For $d \leq 8$, the Strichartz estimate (3.4) with the $B$-admissible exponent $\left(\tilde{q}_{1}, 4\right)$ yields

$$
\|\Phi(E, \mathcal{N})\|_{S} \lesssim\|(\operatorname{Re} \mathcal{N}) E\|_{L_{T}^{\tilde{q}_{1}^{\prime}} L_{x}^{\frac{4}{3}}} \leq T^{1-\frac{d}{8}}\|\mathcal{N}\|_{L_{T}^{\infty} L^{2}}\|E\|_{L_{T}^{\tilde{q}_{1} L_{x}^{4}}} \lesssim T^{1-\frac{d}{8}}\|\mathcal{N}\|_{W}\|E\|_{S},
$$

where we used the Sobolev embedding $B_{2\{-d / 8\}, 2}^{d / 8} \subset L^{2\{-d / 4\}}=L^{4}$ to the norm of $E$. Since $e^{-i t \mathrm{H}}$ is $L_{x}^{2}$-preserving, we have

$$
\|\Psi(E)\|_{W} \leq\left\|\mathrm{U}|E|^{2}\right\|_{L_{T}^{1} L_{x}^{2}} \lesssim\|E\|_{L_{T}^{2} L_{x}^{4}}^{2} \leq T^{1-d / 8}\|E\|_{L_{T}^{\tilde{q}_{1}} L_{x}^{4}} \lesssim T^{1-d / 8}\|E\|_{S}^{2} .
$$

The difference is estimated in the same way. Hence the map $\Lambda$ is a contraction on the closed ball

$$
X_{M}:=\left\{(E, \mathcal{N}) \in S \times W \mid\|(E, \mathcal{N})\|_{S \times W} \leq M\right\}
$$

if $1 \leq d \leq 7$, and we choose $M>0$ large enough and then $T>0$ small enough such that

$$
\left\|E_{0}\right\|_{L_{x}^{2}}+\left\|\mathcal{N}_{0}\right\|_{L_{x}^{2}} \ll M \ll T^{d / 8-1} .
$$

In the case of $d=8$, we cannot gain any positive power of $T$ from the Strichartz estimate as above, since it is the $L^{2}$-critical dimension for the bi-harmonic Schrödinger equation with the quadratic power nonlinearity. Hence we need to use decay of the free solutions in the Strichartz norms as $T \rightarrow+0$, for which the $L_{T}^{\infty} L_{x}^{2}$ norm in (4.5) has to be replaced. For example, we have

$$
\|\Phi(E, \mathcal{N})\|_{S} \lesssim\|(\operatorname{Re} \mathcal{N}) E\|_{L_{T}^{2} L_{x}^{4 / 3}} \leq\|\mathcal{N}\|_{L_{T}^{\infty} L^{2}+L_{T}^{4} L_{x}^{8 / 3}}\|E\|_{L_{T}^{2} L_{x}^{4} \cap L_{T}^{4} L_{x}^{8 / 3}}
$$

Note that $(2,4)=(2,2\{-2\})$ and $(4,8 / 3)$ are $B$-admissible. In particular $W \not \subset$ $L_{T}^{4} L_{x}^{8 / 3}$, but we have

$$
\left\|e^{-i t \mathrm{H}} f\right\|_{L_{T}^{\infty} L_{x}^{2}+L_{T}^{4} L_{x}^{8 / 3}} \rightarrow 0 \quad(T \rightarrow+0)
$$

for any $f \in L^{2}\left(\mathbb{R}^{8}\right)$. To see this, for any $\epsilon>0$, take some $g \in H^{1 / 2}\left(\mathbb{R}^{8}\right)$ such that $\|f-g\|_{L_{x}^{2}}<\epsilon$. The Strichartz estimate (3.6), together with the Sobolev embedding, implies that

$$
\left\|e^{-i t \mathrm{H}} g\right\|_{L_{T}^{4} L_{x}^{8 / 3}} \lesssim\left\|e^{-i t \mathrm{H}} g\right\|_{L_{T}^{4} B_{2\{1 / 2\}, 2}^{1 / 2}} \lesssim\|g\|_{H^{1 / 2}}<\infty
$$

so it tends to 0 as $T \rightarrow+0$, implying that

$$
\limsup _{T \rightarrow+0}\left\|e^{-i t \mathrm{H}} f\right\|_{L_{T}^{\infty} L_{x}^{2}+L_{T}^{4} L_{x}^{8 / 3}} \leq \epsilon .
$$

Since $\epsilon>0$ is arbitrary, the left side must be 0 . Hence all the norms in the right side of (4.9) and in the middle of (4.6) $\left(L_{T}^{\tilde{q}} L_{x}^{4}=L_{T}^{2} L_{x}^{4}\right)$ are decaying as $T \rightarrow+0$ for the free solutions $E_{\mathrm{F}}$ and $\mathcal{N}_{\mathrm{F}}$. Hence $\Lambda$ is a contraction on the closed and absolutely convex subset

$$
\tilde{X}_{\delta}:=\left\{(E, \mathcal{N}) \in S \times W \mid\|E\|_{L_{T}^{2} L_{x}^{4} \cap L_{T}^{4} L_{x}^{8 / 3}}+\|\mathcal{N}\|_{L_{T}^{\infty} L^{2}+L_{T}^{4} L_{x}^{8 / 3}} \leq \delta\right\}
$$


for $d=8$ and some small absolute constant $\delta>0$, provided that $T>0$ is small enough such that $\left(E_{\mathrm{F}}, \mathcal{N}_{\mathrm{F}}\right) \in \tilde{X}_{\delta / 2}$. Note that $X_{\delta} \subset \tilde{X}_{\delta}$, but $T$ cannot be estimated in terms of the initial norm.

Thus for $d \leq 8$ and for all initial data in $L^{2}\left(\mathbb{R}^{d}\right)$, we have obtained the unique local solution in some subset of $S \times W$ for some time interval [0,T]. The continuity of the solution map and the uniqueness of solutions in $S \times W$ are both proven in the standard way by using the same estimates on difference. This concludes the local well-posedness of (1.6) in $L^{2}\left(\mathbb{R}^{d}\right)$ for $d \leq 8$.

\section{Global Well-Posedness for the $Q Z$ System}

Next we prove the global well-posedness for $d \leq 5$, which is the latter part of Theorem 1.1. Since we have the local well-posedness in $L^{2}\left(\mathbb{R}^{d}\right)$, the standard argument yields conservation of $\|E(t)\|_{L_{x}^{2}}=\|E(0)\|_{L_{x}^{2}}<\infty$ for the $L^{2}$ solutions obtained above. Moreover, since we have a lower bound on the existence time $T>0$ as in (4.8) in terms of the initial $L^{2}$ norm (for $d \leq 7$ ), it suffices to derive an a priori bound on $\|N(t)\|_{L^{2}}$ in terms of $t>0$ and

$$
m_{0}:=\left\|E_{0}\right\|_{L^{2}}=\|E(t)\|_{L^{2}}, \quad n_{0}:=\left\|\mathcal{N}_{0}\right\|_{L^{2}} .
$$

Let $(E, \mathcal{N})$ be a local solution with the initial data $\left(E_{0}, \mathcal{N}_{0}\right) \in L^{2}\left(\mathbb{R}^{d}\right)^{2}$, and let

$$
E^{1}:=\Phi(E, \mathcal{N})=E-E_{\mathrm{F}}, \quad \mathcal{N}^{1}:=\Psi(E)=\mathcal{N}-\mathcal{N}_{\mathrm{F}}
$$

denote the inhomogeneous part of the solutions. The goal is to bound $\mathcal{N}^{1}$ in $L_{x}^{2}$. It is particularly easy in $d=1$, since the Strichartz estimate (3.6) yields

$$
\left\|\mathcal{N}^{1}\right\|_{W} \lesssim\left\|\mathrm{U}^{-1 / 4+1}|E|^{2}\right\|_{L_{T}^{4 / 3} L_{x}^{1}} \lesssim T^{3 / 4}\|E\|_{L_{T}^{\infty} L_{x}^{2}}=T^{3 / 4} m_{0}^{2} .
$$

For $d \geq 2$, we cannot close such estimates by using solely $\|E\|_{L_{T}^{\infty} L_{x}^{2}}$, but we need some other Strichartz norms which are not a priori bounded. However, we can still get an a priori bound, as long as the order of the other norms is less than 1 and we have a positive power of the time interval $T>0$. More precisely, we have

Lemma 3. Let $1 \leq d \leq 6$. Then for some constants $\mathrm{a} \geq 0, \mathrm{~b}>0$, and $\mathrm{c} \in[0,1]$, we have

$$
\left\|E^{1}\right\|_{S} \lesssim T^{\mathrm{a}} m_{0}\|\mathcal{N}\|_{W}, \quad\left\|\mathcal{N}^{1}\right\|_{W} \lesssim T^{\mathrm{b}} m_{0}^{2-\mathrm{c}}\|E\|_{S}^{\mathrm{c}},
$$

where the implicit constants depend only on c. Specifically, we can take

$$
\mathrm{a}=\left\{\begin{array}{ll}
(4-d) / 4 & (d \leq 4) \\
0 & (d \geq 5)
\end{array} ; \quad \mathrm{b}=\left\{\begin{array}{ll}
3 / 4 & (d=1) \\
(6-d) / 8 & (d \geq 2)
\end{array} ; \quad \begin{cases}\mathrm{c}=0 \\
\mathrm{c}>0 \\
\mathrm{c}=(d-2) / 4 & (d \geq 3)\end{cases}\right.\right.
$$

The above specific values of a, b, c seem to be optimal (for $m_{0}, n_{0}, T \rightarrow \infty$ ) as far as our argument can reach.

Proof of Lemma 3. First consider the estimate on $E^{1}$ using the Strichartz estimate (3.4). For $d \leq 4$,

$$
\left\|E^{1}\right\|_{S} \lesssim\|(\operatorname{Re} \mathcal{N}) E\|_{L_{T}^{q_{1}^{\prime} L_{x}^{4 / 3}}} \leq T^{1-2 / q_{1}}\|\mathcal{N}\|_{L_{T}^{q_{1}} L_{x}^{4}}\|E\|_{L_{T}^{\infty} L_{x}^{2}} \leq T^{\mathrm{a}} m_{0}\|\mathcal{N}\|_{W} .
$$

For $5 \leq d \leq 6$, using the Sobolev embedding $L^{2\{(8-d) / 2\}} \subset B_{2\{1\}, 2}^{-1}$ as well, we obtain

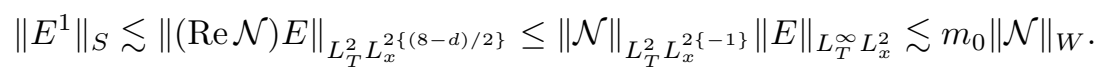


Next consider the estimate on $\mathcal{N}^{1}$ using the Strichartz estimate (3.6). The case $d=1$ was already done in (5.1). For $d=2$ we obtain, for any c $\in(0,1]$ and with implicit constants depending on c,

$$
\begin{aligned}
\left\|\mathcal{N}^{1}\right\|_{W} & \lesssim\left\||E|^{2}\right\|_{\left[L_{T}^{2} L_{x}^{1}, L_{T}^{1} L_{x}^{2}\right]_{\mathrm{c} \theta}} \leq T^{1 / 2}\|E\|_{L_{T}^{\infty} L_{x}^{2}}\|E\|_{\left[L_{T}^{\infty} L_{x}^{2}, L_{T}^{2} L_{x}^{\infty}\right]_{\mathrm{c} \theta}} \\
& \lesssim T^{1 / 2} m_{0}^{2-\mathrm{c}}\|E\|_{S}^{\mathrm{c}} .
\end{aligned}
$$

For $3 \leq d \leq 6$ we obtain, using $\mathrm{b}=1 / 2-1 / \tilde{q}_{2}=(6-d) / 8$,

$$
\left\|\mathcal{N}^{1}\right\|_{W} \lesssim\left\||E|^{2}\right\|_{L_{T}^{2} L_{x}^{2\{1\}}} \leq T^{\mathrm{b}}\|E\|_{L_{T}^{\infty} L_{x}^{2}}\|E\|_{L_{T}^{\tilde{q}_{2} L_{x}^{d}}} .
$$

The last norm is further estimated by the complex interpolation and the Sobolev embedding

$$
\left[L_{T}^{\infty} L^{2}, L_{T}^{2} B_{2\{-1\}, 2}^{1}\right]_{2 / \tilde{q}_{2}}=L_{T}^{\tilde{q}_{2}} B_{2\left\{-2 / \tilde{q}_{2}\right\}, 2}^{2 / \tilde{q}_{2}} \subset L_{T}^{\tilde{q}_{2}} L_{x}^{d} .
$$

Injecting the corresponding inequality into the above yields the desired estimate on $\mathcal{N}^{1}$.

A priori bounds follow immediately from Lemma 3 for $d \leq 5$ :

$$
\begin{aligned}
\|\mathcal{N}\|_{W} & \lesssim\left\|\mathcal{N}_{0}\right\|_{L_{x}^{2}}+\left\|\mathcal{N}^{1}\right\|_{W} \lesssim n_{0}+T^{\mathrm{b}} m_{0}^{2-\mathrm{c}}\|E\|_{S}^{\mathrm{c}} \\
& \lesssim n_{0}+T^{\mathrm{b}} m_{0}^{2-\mathrm{c}}\left(\left\|E_{0}\right\|_{L_{x}^{2}}+\left\|E^{1}\right\|_{S}\right)^{\mathrm{c}} \lesssim n_{0}+T^{\mathrm{b}} m_{0}^{2}+T^{\mathrm{b}+\mathrm{ac}} m_{0}^{2}\|\mathcal{N}\|_{W}^{\mathrm{c}} \\
& \lesssim n_{0}+T^{\mathrm{b}} m_{0}^{2}+\left(\epsilon^{-1} T^{\mathrm{b}+\mathrm{ac}} m_{0}^{2}\right)^{1 /(1-\mathrm{c})}+\epsilon\|\mathcal{N}\|_{W},
\end{aligned}
$$

where the last step used Young's inequality for arbitrary $\epsilon>0$. Choosing $\epsilon$ appropriately small, the last term is absorbed by the left side. Thus we obtain

$$
\|\mathcal{N}\|_{W} \lesssim n_{0}+T^{\mathrm{b}} m_{0}^{2}+T^{\frac{\mathrm{b}+\mathrm{ac}}{1-\mathrm{c}}} m_{0}^{\frac{2}{1-\mathrm{c}}} .
$$

Injecting the specific values of a, b, c, we obtain, for any $0<\epsilon<1 / 2$,

$$
\begin{aligned}
& d=1 \Longrightarrow\|\mathcal{N}\|_{W} \lesssim n_{0}+T^{3 / 4} m_{0}^{2}, \\
& d=2 \Longrightarrow\|\mathcal{N}\|_{W} \lesssim n_{0}+T^{1 / 2} m_{0}^{2}\left[1+\left(T m_{0}^{2}\right)^{\epsilon}\right], \\
& d=3 \Longrightarrow\|\mathcal{N}\|_{W} \lesssim n_{0}+T^{3 / 8} m_{0}^{2}+T^{7 / 12} m_{0}^{8 / 3}, \\
& d=4 \Longrightarrow\|\mathcal{N}\|_{W} \lesssim n_{0}+T^{1 / 4} m_{0}^{2}+\left[T^{1 / 4} m_{0}^{2}\right]^{2+\epsilon}, \\
& d=5 \Longrightarrow\|\mathcal{N}\|_{W} \lesssim n_{0}+T^{1 / 8} m_{0}^{2}+\left[T^{1 / 8} m_{0}^{2}\right]^{4} .
\end{aligned}
$$

Thus we obtain the global well-posedness for $d \leq 5$, thereby concluding the proof of Theorem 1.1. Note that the above argument does not work in $d=6$ since $\mathrm{a}=\mathrm{b}=0$ and $\mathrm{c}=1$.

\section{Small Data SCATtering of The $Q Z$ System}

Now we prove Theorem 1.2, namely, the small data scattering in $L^{2}\left(\mathbb{R}^{d}\right)$ for $4 \leq d \leq 8$. It suffices to derive a set of estimates on the Duhamel integrals $\Phi(E, \mathcal{N})$ and $\Psi(E)$ (see (4.1) and (4.2) ) by the Strichartz norms that are uniform with respect to $T \in(0, \infty]$.

Proof of Theorem 1.2. Let $4 \leq d \leq 8$. Using the Strichartz estimate (3.4) together with the Sobolev embeddings $L^{2\{s\}} \subset B_{2\{1\}, 2}^{-1}$ and $B_{2\{-1\}, 2}^{1} \subset L^{2\{-s\}}$ for $1 \leq s \leq 2$, we obtain

$$
\|\Phi(E, \mathcal{N})\|_{S} \lesssim\|(\operatorname{Re} \mathcal{N}) E\|_{L_{T}^{2} L_{x}^{2\{d / 4\}}} \leq\|\mathcal{N}\|_{L_{T}^{\infty} L_{x}^{2}}\|E\|_{L_{T}^{2} L_{x}^{2\{-d / 4\}}} \lesssim\|\mathcal{N}\|_{W}\|E\|_{S}
$$


Since $e^{-i t \mathrm{H}}$ is $L_{x}^{2}$-preserving, we have

$$
\|\Psi(E)\|_{W} \lesssim\left\||E|^{2}\right\|_{L_{T}^{1} L_{x}^{2}}=\|E\|_{L_{T}^{2} L_{x}^{2\{-d / 4\}}}^{2} \lesssim\|E\|_{S}^{2}
$$

The difference is estimated in the same way. Hence we can choose both $\left\|E_{0}\right\|_{L^{2}}+$ $\left\|\mathcal{N}_{0}\right\|_{L^{2}}$ and $M$ to be sufficiently small such that the iteration map $\Lambda$ is a contraction on $X_{M}$ defined in (4.7) uniformly for all $T \in(0, \infty]$. Thus we obtain a unique global solution $(E, \mathcal{N})$ as the unique fixed point of $\Lambda$, with the global Strichartz bound

$$
\|E\|_{L_{t \in \mathbb{R}}^{\infty} L_{x}^{2} \cap L_{t \in \mathbb{R}}^{2} B_{2\{-1\}, 2}^{1}}+\|\mathcal{N}\|_{L_{t \in \mathbb{R}}^{\infty} L_{x}^{2} \cap L_{t \in \mathbb{R}}^{2} L_{x}^{2\{-1\}}} \lesssim\left\|E_{0}\right\|_{L^{2}}+\left\|\mathcal{N}_{0}\right\|_{L^{2}}(\ll 1) .
$$

For the scattering result, the Strichartz estimate also implies, for any $T_{1}<T_{2}$, that

$$
\begin{aligned}
\left\|\left[e^{i t \mathrm{H}^{2}} E(t)\right]_{T_{1}}^{T_{2}}\right\|_{L_{x}^{2}} & =\left\|\int_{T_{1}}^{T_{2}} e^{-i s \mathrm{H}^{2}}((\operatorname{Re} \mathcal{N}) E)(s) d s\right\|_{L_{x}^{2}} \\
& \lesssim\|(\operatorname{Re} \mathcal{N}) E\|_{L_{t \in\left(T_{1}, T_{2}\right)}^{2} L_{x}^{2\{d / 4\}}} \\
& \leq\|\mathcal{N}\|_{L_{t \in \mathbb{R}}^{\infty} L_{x}^{2}}\|E\|_{L_{t \in\left(T_{1}, T_{2}\right)}^{2}} L_{x}^{2\{-d / 4\}} .
\end{aligned}
$$

Since the last norm is vanishing as $T_{1} \rightarrow \infty$ or $T_{2} \rightarrow-\infty$, we deduce that $e^{i t \mathrm{H}^{2}} E(t)$ is convergent in $L_{x}^{2}$ as $t \rightarrow \pm \infty$, namely, the scattering of $E(t)$ in $L^{2}$. The scattering of $\mathcal{N}(t)$ is obtained similarly.

\section{ACKNOWLEDGMENT}

The first author wants to thank Satoshi Masaki at Osaka University, Yoshio Tsutsumi at Kyoto University, and Takayoshi Ogawa at Tohoku University for their inspiring conversations and discussions, including the hospitality from their institutions.

\section{REFERENCES}

[1] J. Bourgain and J. Colliander, On wellposedness of the Zakharov system, Internat. Math. Res. Notices 11 (1996), 515-546, DOI 10.1155/S1073792896000359. MR1405972

[2] I. Bejenaru, S. Herr, J. Holmer, and D. Tataru, On the 2D Zakharov system with $L^{2}$-Schrödinger data, Nonlinearity 22 (2009), no. 5, 1063-1089, DOI 10.1088/0951$7715 / 22 / 5 / 007$. MR 2501036

[3] Matania Ben-Artzi, Herbert Koch, and Jean-Claude Saut, Dispersion estimates for fourth order Schrödinger equations (English, with English and French summaries), C. R. Acad. Sci. Paris Sér. I Math. 330 (2000), no. 2, 87-92, DOI 10.1016/S0764-4442(00)00120-8. MR1745182

[4] Thierry Cazenave, Semilinear Schrödinger equations, Courant Lecture Notes in Mathematics, vol. 10, New York University, Courant Institute of Mathematical Sciences, New York; American Mathematical Society, Providence, RI, 2003. MR2002047

[5] Tsai-Jung Chen, Yung-Fu Fang, and Kuan-Hsiang Wang, Low regularity global well-posedness for the quantum Zakharov system in 1D, Taiwanese J. Math. 21 (2017), no. 2, 341-361, DOI $10.11650 / \mathrm{tjm} / 7806$. MR 3632519

[6] James Colliander, Justin Holmer, and Nikolaos Tzirakis, Low regularity global well-posedness for the Zakharov and Klein-Gordon-Schrödinger systems, Trans. Amer. Math. Soc. 360 (2008), no. 9, 4619-4638, DOI 10.1090/S0002-9947-08-04295-5. MR2403699

[7] Yung-Fu Fang, Hsi-Wei Shih, and Kuan-Hsiang Wang, Local well-posedness for the quantum Zakharov system in one spatial dimension, J. Hyperbolic Differ. Equ. 14 (2017), no. 1, 157192, DOI 10.1142/S0219891617500059. MR3625889

[8] L. G. Garcia, F. Haas, L. P. L. de Oliveira, and J. Goedert, Modified Zakharov equations for plasmas with a quantum correction, Phys. Plasmas 12 (2005), 012302. 
[9] L. Glangetas and F. Merle, Existence of self-similar blow-up solutions for Zakharov equation in dimension two. I, Comm. Math. Phys. 160 (1994), no. 1, 173-215. MR.1262194

[10] L. Glangetas and F. Merle, Concentration properties of blow-up solutions and instability results for Zakharov equation in dimension two. II, Comm. Math. Phys. 160 (1994), no. 2, 349-389. MR.1262202

[11] Stephen Gustafson, Kenji Nakanishi, and Tai-Peng Tsai, Scattering for the Gross-Pitaevskii equation, Math. Res. Lett. 13 (2006), no. 2-3, 273-285, DOI 10.4310/MRL.2006.v13.n2.a8. MR.2231117

[12] Zihua Guo, Lizhong Peng, and Baoxiang Wang, Decay estimates for a class of wave equations, J. Funct. Anal. 254 (2008), no. 6, 1642-1660, DOI 10.1016/j.jfa.2007.12.010. MR2396016

[13] J. Ginibre, Y. Tsutsumi, and G. Velo, On the Cauchy problem for the Zakharov system, J. Funct. Anal. 151 (1997), no. 2, 384-436, DOI 10.1006/jfan.1997.3148. MR1491547

[14] J. Ginibre and G. Velo, Smoothing properties and retarded estimates for some dispersive evolution equations, Comm. Math. Phys. 144 (1992), no. 1, 163-188. MR 1151250

[15] J. Ginibre and G. Velo, Generalized Strichartz inequalities for the wave equation, J. Funct. Anal. 133 (1995), no. 1, 50-68, DOI 10.1006/jfan.1995.1119. MR.1351643

[16] Yanfeng Guo, Jingjun Zhang, and Boling Guo, Global well-posedness and the classical limit of the solution for the quantum Zakharov system, Z. Angew. Math. Phys. 64 (2013), no. 1, 53-68, DOI 10.1007/s00033-012-0215-y. MR3023075

[17] Fernando Haas, Quantum plasmas: An hydrodynamic approach, Springer Series on Atomic, Optical, and Plasma Physics, vol. 65, Springer, New York, 2011. MR3024749

[18] F. Haas and P. K. Shukla, Quantum and classical dynamics of Langmuir wave packets. Rev. E 79 (2009), 066402.

[19] Markus Keel and Terence Tao, Endpoint Strichartz estimates, Amer. J. Math. 120 (1998), no. 5, 955-980. MR 1646048

[20] Carlos E. Kenig, Gustavo Ponce, and Luis Vega, Oscillatory integrals and regularity of dispersive equations, Indiana Univ. Math. J. 40 (1991), no. 1, 33-69, DOI 10.1512/iumj.1991.40.40003. MR.1101221

[21] Carlos E. Kenig, Gustavo Ponce, and Luis Vega, On the Zakharov and Zakharov-Schulman systems, J. Funct. Anal. 127 (1995), no. 1, 204-234, DOI 10.1006/jfan.1995.1009. MR.1308623

[22] J.-L. Lions, Quelques méthodes de résolution des problèmes aux limites non linéaires (French), Dunod; Gauthier-Villars, Paris, 1969. MR0259693

[23] Tohru Ozawa and Yoshio Tsutsumi, Existence and smoothing effect of solutions for the Zakharov equations, Publ. Res. Inst. Math. Sci. 28 (1992), no. 3, 329-361, DOI $10.2977 /$ prims/1195168430. MR 1184829

[24] Tohru Ozawa and Yoshio Tsutsumi, The nonlinear Schrödinger limit and the initial layer of the Zakharov equations, Differential Integral Equations 5 (1992), no. 4, 721-745. MR1167491

[25] Benoit Pausader, Global well-posedness for energy critical fourth-order Schrödinger equations in the radial case, Dyn. Partial Differ. Equ. 4 (2007), no. 3, 197-225, DOI 10.4310/DPDE.2007.v4.n3.a1. MR2353631

[26] Elias M. Stein, Harmonic analysis: real-variable methods, orthogonality, and oscillatory integrals, with the assistance of Timothy S. Murphy, Monographs in Harmonic Analysis, III, Princeton Mathematical Series, vol. 43, Princeton University Press, Princeton, NJ, 1993. MR 1232192

[27] V. E. Zakharov, Collapse of Langmuir waves, Sov. Phys. JETP 35 (1972), 908-914.

Department of Mathematics, National Cheng Kung University, No. 1, Dasyue Road,

TAINAN City 70101, TAIWAN

Email address: yffang@mail.ncku.edu.tw

Research Institute for Mathematical Sciences, Kyoto University, Kyoto 606-8502, JAPAN

Email address: kenji@kurims.kyoto-u.ac.jp 